\title{
Variability in the nutraceutical properties of fenugreek (Trigonella foenum-graecum L.) seeds
}

\section{Variabilidad de las propiedades nutracéuticas de la alholva (Trigonella foenum-graecum L.)}

KRISHAN PAL SINGH', 4

BEENA NAIR ${ }^{2}$

PRAMOD KUMAR JAIN ${ }^{2}$

AJAY KUMAR NAIDU2

SEEMA PAROHA ${ }^{3}$
Variability in seeds size and colour of different genotypes of fenugreek.

Foto: K.P. Singh

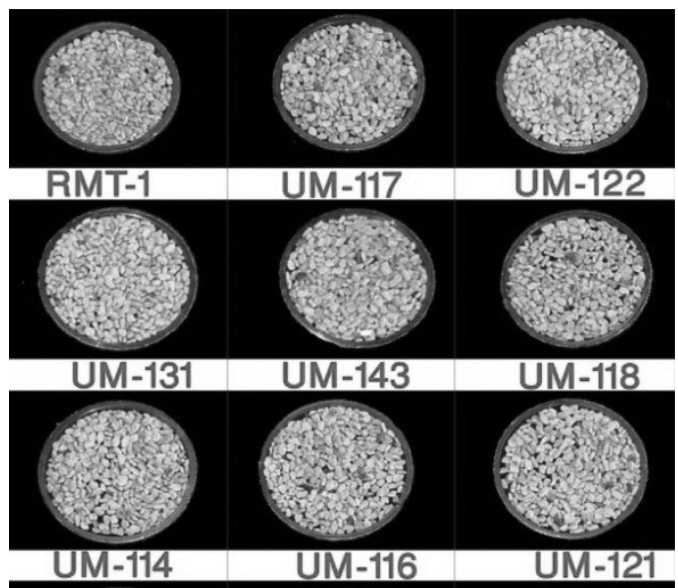

\section{ABSTRACT}

Fenugreek (Trigonella foenum-graecum L.) leaves and seeds have been used extensively for medicinal purposes. Fenugreek seed is known to exhibit anti-diabetic properties and effects such as hypocholesterolaemic, anticancerous and thyroxine-induced hyperglycaemia. The result of the present experiment reveals considerable variability among fenugreek genotypes. They differ in morphology, growth habit, biomass and seed production capability. Chemical constituents of the seed, e.g. polyphenol, phytic acid, saponin, carbohydrate, protein and proximate analysis (moisture \%, ash, fiber, $\mathrm{Zn}, \mathrm{Fe}, \mathrm{Mn}$, and $\mathrm{Mg}$ ) contents also differed markedly. This variability is most often overlooked or underestimated in clinical trials. Our research suggests that the genetic variability and the genotype by environmental interaction will play a significant role when the crop is used by the nutraceutical industry. Our research results indicate that the variability for important traits in fenugreek have a genetic base, making selection for improved levels of these traits possible. Fenugreek plays a major role to progress towards the exploitation of crop to overcome micronutrient deficiency in human beings. The associated issues to strengthen crop biofortification through fenugreek research and development have been dealt in this piece of research work.

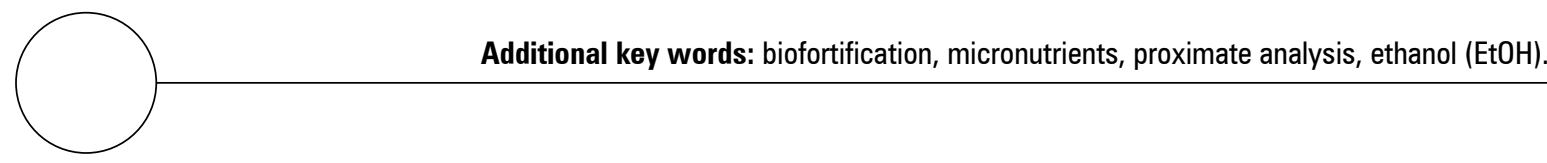

\footnotetext{
Krishi Vigyan Kendra (KVK), Banasthali University, Tonk-304022, Rajasthan (India).

Department of Horticulture, JNKVV, Jabalpur (M.P.), India - 482004, Jabalpur (India).

Department of Biochemistry, JNKVV, Jabalpur (M.P.), India.

4 Corresponding author. drkpsingh2010@gmail.com
} 


\section{RESUMEN}

Las hojas y semillas de alholva o fenogreco (Trigonella foenum-graecum L.) se han utilizado ampliamente con fines medicinales. Se conoce que las semillas de alholva presentan propiedades antidiabéticas y efectos tales como hipocolesterolémico, anti-cancerígeno y tiroxina inducida de la hiperglucemia. Los resultados de los experimentos realizados revelan una considerable variabilidad entre los genotipos de alholva. Se diferencian en la morfología, hábito de crecimiento, biomasa, y capacidad de producción de semillas. Los componentes químicos de la semilla como por ejemplo los contenidos de polifenol, ácido fítico, saponina, carbohidratos, proteínas y análisis proximal (\% de humedad, cenizas, fibra, Zn, Fe, Mn, y Mg) también diferían notablemente. Esta variabilidad es a menudo pasado por alto o es subestimada en los ensayos clínicos. Los resultados sugieren que la variabilidad genética y la interacción genotipo x ambiente desempeñarán un papel importante cuando el cultivo es utilizado por la industria nutracéutica. Además la variabilidad de rasgos importantes de la alholva indican que su base genética, permite la selección para el mejoramiento de los niveles de estos rasgos. La alholva juega un papel importante para avanzar hacia la explotación de su cultivo y superar la deficiencia de micronutrientes en los seres humanos. Los temas asociados para fortalecer la biofortificación de cultivos a través de la investigación y desarrollo de la alholva, serán tratados en esta publicación.

Palabras clave adicionales: biofortificación, micronutrientes, análisis proximal, etanol (EtOH).

Fecha de recepción: 11-09-2013

Aprobada para publicación: 31-10-2013
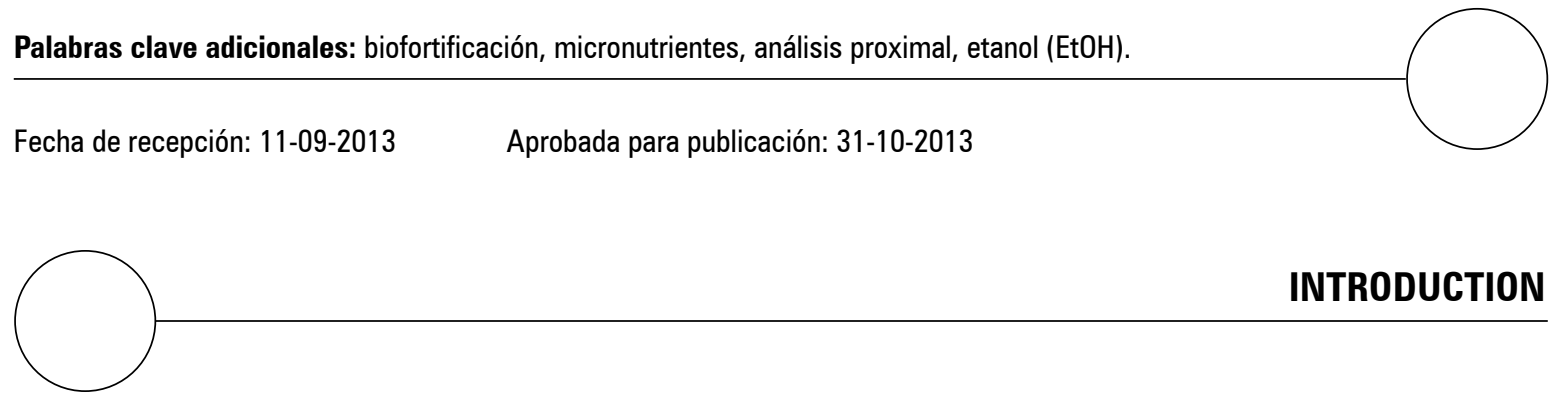

INTRODUCTION

Fenugreek (Trigonella foenum-graecum L.), wild or cultivated, is widely distributed throughout the world. It is an important leafy vegetable cum seed spice with medicinal properties belonging to the sub family Papilionaceae of the family Fabaceae. According to the National Center for Complementary and Alternative Medicine, fenugreek was first mentioned in "an Egyptian papyrus" dating back to 1500 B.C. The generic name, Trigonella, comes from Latin meaning 'little triangle', in reference to the triangular shape of the small yellowish-white flowers. The biological and pharmacological actions of fenugreek are attributed to the variety of constituents, such as steroids, N-compounds, polyphenolic substances, volatile constituents, and amino acids. Fresh or dried fenugreek leaves and its tender stems are edible.

Each $100 \mathrm{~g}$ of leaves contains $86 \%$ moisture, $4.4 \%$ protein, $1 \%$ lipids, $1 \%$ fiber, $395 \mathrm{mg} \mathrm{Ca}$,
$67 \mathrm{mg} \mathrm{Mg}, 51 \mathrm{mg}$ P, $16.5 \mathrm{mg} \mathrm{Fe}, 76 \mathrm{mg} \mathrm{Na}, 31$ mg K, $260 \mathrm{mg} \mathrm{Cu}, 167 \mathrm{mg} \mathrm{S}, 165 \mathrm{mg} \mathrm{Cl}, 2.3 \mathrm{mg}$ carotene (mainly beta, 329 IU Vit. A), $40 \mathrm{mg}$ thiamine, $310 \mathrm{mg}$ riboflavin, $800 \mathrm{mg}$ nicotinic acid, and $52 \mathrm{mg}$ vitamin C; with traces of vitamin $\mathrm{K}$ and high amounts of choline $(13.5 \mathrm{mg}$ $\left.\mathrm{g}^{-1}\right)$. Fenugreek seeds contain $45-60 \%$ carbohydrates, $20-30 \%$ proteins, $5-10 \%$ fixed oils (lipids), pyridine alkaloids, flavonoids, free amino acids, calcium and iron, saponins (0.6-1.7\%), glycosides yielding steroidal sapogenins on hydrolysis, cholesterol and sitosterol, vitamin A, $\mathrm{B}_{1}, \mathrm{C}$ and nicotinic acids and $0.015 \%$ volatile oils (Budavari, 1996; Newall et al., 1996; Mehrafarin et al., 2010).

Crop biofortification is a sustainable and cost effective strategy to tackle malnutrition in developing countries. Fenugreek is best known for presence of the distinctive, pungent aromatic compounds in the seed (Max, 1992) that im- 
parts flavour, colour and aroma to foods, making it a highly desirable supplement for culinary applications. Majority of the Indian population belongs to vegetarian class. In such a situation, a leafy vegetable, such as fenugreek, is of utmost importance due to its high nutritive value, medicinal importance, and industrial uses. Recent researches (Nasroallah and Moradi, 2013) have identified fenugreek as a valuable medicinal plant with a potential for multipurpose uses and also as a source for preparing raw materials of pharmaceutical industry, especially steroidal hormones. The main objective of this study was to widen the knowledge of the composition of various biochemical contents in fenugreek seed, which could overcome some micronutrient deficiencies in human beings. Therefore, the content and composition of proteins, carbohydrates, steroid saponins, polyphenols, phytic acid and proximate analysis in different fenugreek genotypes were investigated. Phytic acid is the primary source of inositol and storage phosphorus in plant seeds. This paper synthesizes the progress towards mounting the knowledge about spice rich in micronutrients by exploiting its natural genetic variability utilizing conventional breeding.

\section{MATERIAL AND METHODS}

The present study was undertaken in Rabi seasons of 2009-2010, 2010-2011, and 2011-2012 at Vegetable Research Farm, Department of Horticulture, Jawaharlal Nehru Krishi Vishwavidyalaya (JNKVV), Jabalpur (Madhya Pradesh), India. The experimental material consisted of 102 diverse genotypes from geographic and genetic origin and three checks namely Pusa Early Bunch, Gujarat Methi-2 and RMT-1 that are locally used and famous high yielding improved varieties. These 102 germplasm lines were evaluated in a randomized block design with three replicates. The experiment was laid in 4 blocks with 26 test entries (germplasm) and 3 checks in each block. The seeds were sown directly in the experimental site. The plot size was of $1.0 \mathrm{~m}$ $\mathrm{x} 10 \mathrm{~m}$ with row to row spacing of $30 \mathrm{~cm}$ and plant to plant spacing of $10 \mathrm{~cm}$.

\section{Soil and climate condition of site}

Before the layout of experimental field, farm yard manure $20 \mathrm{t} \mathrm{ha}^{-1}$ was mixed well in soil. The chemical fertilizers were applied manually at the time of sowing. FYM was applied $20 \mathrm{t}$ $\mathrm{ha}^{-1}$ and mixed uniformly in soil at the time of last preparation of field. Nitrogen and phosphorus were given in the form of urea and potash through muriate of potash@ 35:70:60 kg ha-1. Half amount of $\mathrm{N}$ with full amount of $\mathrm{P}$ and $\mathrm{K}$ were given per plot as basal dose and rest amount of $\mathrm{N}$ was given as top dressing after 40 days of sowing. The standard agronomic practices were adopted for normal crop growth.

Jabalpur is situated at $23.900 \mathrm{~N}$ latitude and 79.580 E longitudes at an altitude of $411.87 \mathrm{~m}$ above the mean sea level. This region has subtropical, semi-arid climate with hot and dry summer and cold winter with occasional showers. The average rainfall is about $1258.4 \mathrm{~mm}$, which is received mostly from July to September. Temperature varies from $60^{\circ} \mathrm{C}$ being minimum in January to $450^{\circ} \mathrm{C}$ being maximum in May and June. This area is under "Kymore plateau and Satpura hills agro-climatic zone" as per norms of National Agricultural Research Programme.

\section{Biochemical parameters of seeds}

The crude protein was estimated by Lowry's Method (Lowry et al., 1951), moisture content, crude fibre and total ash were determined as described in the AOAC (1995), total carbohydrates were estimated by Anthrone Method (Hedge and Hofreiter, 1962), steroid saponins by the method elaborated by Lin and Yang (2008), and phenols as given by Malik and Singh (1980). 


\section{Antinutritional factor}

The antinutritional factor (phytic acid) was studied according to Wheeler and Ferrel (1971). Weigh a finally ground (40 mesh) sample estimated to contain 5 to $30 \mathrm{mg}$ phytate $P$ in to a 125 $\mathrm{mL}$ Erlenmeyer flask. Extract in $50 \mathrm{~mL} 3 \% \mathrm{TCA}$ for $30 \mathrm{~min}$ with mechanical shaking or with occasional swirling by hand for $45 \mathrm{~min}$. Centrifuge the suspension and transfer a $10 \mathrm{~mL}$ aliquot of the supernatant to a $40 \mathrm{~mL}$ conical centrifuge tube. Add $4 \mathrm{~mL}$ of $\mathrm{FeCl}_{3}$ solution to the aliquot by blowing rapidly from the pipette. Heat the contents in a boiling water bath for $45 \mathrm{~min}$. if the supernatant is not clear after $30 \mathrm{~min}$, add one or two drops of $3 \%$ sodium sulphate in $3 \%$ TCA and continue heating. Centrifuge (10 to 15 $\mathrm{min}$ ) and carefully decant the clear supernatant. Wash the precipitate twice by dispersing well in 20 to $25 \mathrm{~mL} 3 \%$ TCA, heat in boiling water for 5 to $10 \mathrm{~min}$. Repeat washing with water. Disperse the precipitate in a few $\mathrm{ml}$ of water and add 3 $\mathrm{mL} 1.5 \mathrm{NaOH}$ with mixing. Bring volume to approximately $30 \mathrm{~mL}$ with water and heat in boiling water for $30 \mathrm{~min}$. Filler hot (quantitatively) through a moderately retentive paper Whatman No. 2. Wash the precipitate with $60-70 \mathrm{~mL}$ hot water and discard the filtrate. Dissolve the precipitate from the paper with $40 \mathrm{~mL}$ hot $3.2 \mathrm{~N}$ $\mathrm{HNO}_{3}$ into a $100 \mathrm{~mL}$ volumetric flask. Wash the paper with several portions of water, collecting the washing in the same flask. Cool flask and contents to room temperature and dilute to volume with water. Transfer a $5 \mathrm{~mL}$ aliquot to another $100 \mathrm{~mL}$ volumetric flask and dilute to approximately $70 \mathrm{~mL}$. Add $20 \mathrm{~mL}$ of $1.5 \mathrm{M} \mathrm{KSCN}$ dilute to volume, and read colour immediately (within $1 \mathrm{~min}$ ) at $480 \mathrm{~nm}$. Run a reagent blank with each set of samples (seeds).

\section{Estimation of micronutrients (atomic absorp- tion spectrophotometer)}

To estimate micronutrient, especially $\mathrm{Fe}, \mathrm{Mg}$, $\mathrm{Cu}, \mathrm{Zn}$, and $\mathrm{Mn}$, contents in the seeds, the mixture was digested with di-acid (nitric acid and perchloric acid in 2:1 ratio) mixture, diluted to $50 \mathrm{~mL}$ with deionized water, and directly run on atomic absorption spectrophotometer. The standard was calibrated on AAS and then samples were read. The concentration reading given by AAS was multiplied by the dilution factor used during digestion.

\section{Statistical analysis}

The data on quantitative characters were statistically analyzed on the basis of model described by Cochran and Cox (1950) for randomized block design. In order to test the significance of treatments, critical difference was computed (Fisher and Yates, 1963).

$$
\mathrm{Y}_{\mathrm{ij}}=\mu+\mathrm{b}_{\mathrm{i}}+\mathrm{t}_{\mathrm{j}}+\mathrm{e}_{\mathrm{ij}}
$$

\section{RESULTS}

The mean performance of different genotypes and their contributing characters is given in table 1. Polyphenol $(\mathrm{mg} / 100 \mathrm{~g})$ content ranged from 95.97 to $147.97 \mathrm{mg} / 100 \mathrm{~g}$ with an overall mean of $107.22 \mathrm{mg} / 100 \mathrm{~g}$ in the seeds. The minimum polyphenol content was observed in NDM-278 (95.50 mg/100 g), followed by HM$260(98.17 \mathrm{mg} / 100 \mathrm{~g})$ whereas the maximum $(148.50 \mathrm{mg} / 100 \mathrm{~g})$ in genotype UM-117.

In the present investigation, phytic acid content ranged between 102.2 and $213.20 \mathrm{mg} / 100 \mathrm{~g}$ with an overall mean of $143.29 \mathrm{mg} / 100 \mathrm{~g}$. The lowest phytic acid content was recorded in the genotype RM-199 (102.2 mg/100 g), whereas, the highest in HM-267 $(213.20 \mathrm{mg} / 100 \mathrm{~g})$.

Saponin content varied from 1,245 to 1,780.67 $\mathrm{mg} / 100 \mathrm{~g}$ with an average of $1,537.96 \mathrm{mg} / 100 \mathrm{~g}$. The highest recovery of saponin was recorded in genotype RM-185 (1780.67 mg/100g) while, the minimum content was observed in NDM-29 $(1,245 \mathrm{mg} / 100 \mathrm{~g})$ followed by ACC-012 $(1,254$ $\mathrm{mg} / 100 \mathrm{~g})$. 


\section{Table 1. Mean performance of different biochemical content and its contributing traits in fenugreek seeds (pooled)}

\begin{tabular}{|c|c|c|c|c|c|c|c|c|c|c|c|c|c|}
\hline \multirow[b]{2}{*}{ S. No. } & \multirow[b]{2}{*}{ Genotypes } & \multirow{2}{*}{$\begin{array}{l}\text { Polyphenol } \\
\text { (mg/100 g) }\end{array}$} & \multirow{2}{*}{$\begin{array}{l}\text { Phytic acid } \\
\text { (mg/100 g) }\end{array}$} & \multirow{2}{*}{$\begin{array}{l}\text { Saponin } \\
(\mathrm{mg} / 100 \mathrm{~g})\end{array}$} & \multirow{2}{*}{$\begin{array}{c}\text { Carbohydrate } \\
(\%)\end{array}$} & \multirow[b]{2}{*}{ Protein (\%) } & \multicolumn{7}{|c|}{ Proximate analysis } \\
\hline & & & & & & & $\begin{array}{c}\text { Moisture } \\
(\%)\end{array}$ & $\begin{array}{l}\text { Ash } \\
(\%)\end{array}$ & $\begin{array}{c}\text { Fiber } \\
(\mathrm{g} / 100 \mathrm{~g})\end{array}$ & $\begin{array}{c}\mathrm{Zn} \\
(\mathrm{mg} / 100 \mathrm{~g})\end{array}$ & $\begin{array}{c}\mathrm{Fe} \\
(\mathrm{mg} / 100 \mathrm{~g})\end{array}$ & $\begin{array}{c}\mathrm{Mn} \\
(\mathrm{mg} / 100 \mathrm{~g})\end{array}$ & $\begin{array}{c}\mathrm{Mg} \\
(\mathrm{mg} / 100 \mathrm{~g})\end{array}$ \\
\hline 1 & UM-117 & 148.50 & 153.20 & 1563.00 & 1.81 & 18.50 & 8.87 & 3.27 & 6.63 & 2.31 & 10.20 & 1.53 & 148.00 \\
\hline 2 & UM-122 & 125.10 & 132.40 & 1642.00 & 1.76 & 18.13 & 9.23 & 3.20 & 6.10 & 2.33 & 8.83 & 1.45 & 183.00 \\
\hline 3 & UM-131 & 112.27 & 124.30 & 1598.33 & 1.43 & 20.10 & 7.80 & 3.20 & 6.00 & 2.71 & 10.60 & 1.56 & 162.00 \\
\hline 4 & UM-143 & 109.30 & 135.20 & 1567.33 & 1.53 & 20.60 & 7.97 & 3.27 & 6.50 & 2.35 & 9.33 & 1.50 & 168.00 \\
\hline 5 & UM-118 & 105.27 & 127.30 & 1652.00 & 1.32 & 18.30 & 8.67 & 3.17 & 6.10 & 2.49 & 9.27 & 1.48 & 172.00 \\
\hline 6 & UM-114 & 105.53 & 167.20 & 1456.00 & 1.24 & 21.70 & 7.67 & 3.00 & 5.60 & 1.92 & 10.30 & 1.60 & 183.00 \\
\hline 7 & UM-116 & 134.00 & 156.30 & 1364.00 & 1.24 & 23.40 & 9.17 & 3.20 & 6.27 & 1.47 & 10.40 & 1.79 & 149.00 \\
\hline 8 & UM-121 & 124.20 & 163.20 & 1652.00 & 1.33 & 21.90 & 8.87 & 3.53 & 6.87 & 1.48 & 9.40 & 1.46 & 168.00 \\
\hline 9 & UM-123 & 113.50 & 145.30 & 1458.00 & 1.35 & 22.27 & 8.00 & 3.40 & 6.60 & 1.68 & 8.40 & 1.58 & 174.00 \\
\hline 10 & UM-120 & 106.27 & 167.28 & 1652.00 & 1.07 & 21.50 & 8.63 & 3.60 & 6.50 & 1.89 & 10.40 & 1.47 & 178.00 \\
\hline 11 & UM-128 & 105.43 & 167.30 & 1387.00 & 1.74 & 23.53 & 9.27 & 3.23 & 6.60 & 2.32 & 9.27 & 1.68 & 156.00 \\
\hline 12 & UM-133 & 104.53 & 156.30 & 1601.33 & 1.78 & 21.40 & 8.23 & 3.40 & 7.20 & 2.45 & 8.40 & 1.45 & 184.00 \\
\hline 13 & UM-132 & 113.63 & 158.30 & 1669.67 & 1.69 & 21.60 & 9.03 & 3.87 & 6.20 & 2.22 & 8.20 & 1.56 & 174.00 \\
\hline 14 & UM-125 & 104.20 & 123.40 & 1763.00 & 1.67 & 21.60 & 8.43 & 3.60 & 6.20 & 2.45 & 8.40 & 1.48 & 189.00 \\
\hline 15 & UM-130 & 110.20 & 145.27 & 1375.00 & 1.45 & 22.30 & 7.57 & 3.20 & 6.40 & 2.44 & 9.60 & 1.51 & 159.00 \\
\hline 16 & UM-138 & 124.00 & 136.30 & 1389.00 & 1.67 & 21.47 & 8.97 & 3.60 & 6.27 & 2.45 & 10.20 & 1.50 & 167.00 \\
\hline 17 & UM-129 & 110.17 & 167.30 & 1567.00 & 1.36 & 18.60 & 8.20 & 3.57 & 6.50 & 2.56 & 9.27 & 1.48 & 172.00 \\
\hline 18 & UM-135 & 98.30 & 124.30 & 1452.00 & 1.68 & 18.60 & 9.13 & 3.58 & 7.50 & 2.45 & 10.20 & 1.76 & 169.00 \\
\hline 19 & UM-113 & 120.27 & 142.37 & 1567.00 & 1.80 & 21.50 & 9.03 & 3.57 & 8.93 & 2.34 & 9.60 & 1.55 & 174.00 \\
\hline 20 & RMT-361 & 110.30 & 156.27 & 1652.00 & 1.36 & 22.10 & 9.90 & 3.20 & 6.40 & 1.64 & 8.40 & 1.52 & 182.67 \\
\hline 21 & UM-144 & 147.97 & 145.30 & 1563.00 & 1.26 & 22.30 & 8.23 & 3.80 & 6.50 & 1.46 & 9.27 & 1.63 & 186.00 \\
\hline 22 & HM-267 & 120.30 & 213.20 & 1475.00 & 1.26 & 19.07 & 8.33 & 3.40 & 6.27 & 1.57 & 10.13 & 1.46 & 175.00 \\
\hline 23 & HM-281 & 105.30 & 143.70 & 1567.00 & 1.37 & 19.27 & 7.83 & 3.23 & 6.50 & 2.34 & 8.60 & 1.62 & 149.00 \\
\hline 24 & HM-279 & 104.40 & 167.53 & 1645.00 & 1.26 & 20.40 & 8.60 & 3.00 & 6.67 & 1.61 & 10.27 & 1.54 & 164.00 \\
\hline 25 & HM-282 & 109.30 & 145.40 & 1465.00 & 1.46 & 22.10 & 9.00 & 3.20 & 7.10 & 1.62 & 10.30 & 1.52 & 172.00 \\
\hline 26 & HM-278 & 98.50 & 156.30 & 1472.00 & 1.56 & 20.30 & 7.67 & 3.20 & 6.40 & 2.46 & 10.60 & 1.38 & 156.00 \\
\hline 27 & HM-271 & 110.20 & 164.30 & 1562.00 & 1.27 & 18.40 & 9.70 & 3.20 & 6.53 & 2.48 & 11.20 & 1.43 & 175.00 \\
\hline 28 & HM-258-1 & 103.27 & 137.50 & 1642.00 & 1.45 & 18.10 & 11.51 & 3.50 & 6.93 & 2.48 & 10.27 & 1.52 & 178.67 \\
\hline 29 & HM-280 & 103.73 & 142.30 & 1558.67 & 1.34 & 18.67 & 8.97 & 3.40 & 6.60 & 2.48 & 9.80 & 1.54 & 162.00 \\
\hline 30 & HM-258 & 104.93 & 135.63 & 1600.33 & 1.45 & 19.77 & 10.17 & 3.40 & 6.80 & 2.46 & 10.80 & 1.56 & 186.00 \\
\hline 31 & HM-280-1 & 104.40 & 156.27 & 1445.00 & 1.67 & 20.27 & 9.93 & 3.40 & 6.33 & 1.68 & 10.20 & 1.48 & 183.00 \\
\hline 32 & HM-259 & 110.20 & 123.40 & 1575.33 & 1.69 & 20.23 & 7.83 & 3.20 & 6.80 & 1.72 & 10.60 & 1.57 & 189.00 \\
\hline
\end{tabular}




\begin{tabular}{|c|c|c|c|c|c|c|c|c|c|c|c|c|c|}
\hline \multirow[b]{2}{*}{ S. No. } & \multirow[b]{2}{*}{ Genotypes } & \multirow[b]{2}{*}{$\begin{array}{l}\text { Polyphenol } \\
\text { (mg/100 g) }\end{array}$} & \multirow[b]{2}{*}{$\begin{array}{l}\text { Phytic acid } \\
\text { (mg/100 g) }\end{array}$} & \multirow[b]{2}{*}{$\begin{array}{l}\text { Saponin } \\
(\mathrm{mg} / 100 \mathrm{~g})\end{array}$} & \multirow[b]{2}{*}{$\begin{array}{c}\text { Carbohydrate } \\
(\%)\end{array}$} & \multirow[b]{2}{*}{ Protein (\%) } & \multicolumn{7}{|c|}{ Proximate analysis } \\
\hline & & & & & & & $\begin{array}{c}\text { Moisture } \\
(\%)\end{array}$ & $\begin{array}{l}\text { Ash } \\
(\%)\end{array}$ & $\begin{array}{c}\text { Fiber } \\
(\mathrm{g} / 100 \mathrm{~g})\end{array}$ & $\begin{array}{c}\mathrm{Zn} \\
(\mathrm{mg} / 100 \mathrm{~g})\end{array}$ & $\begin{array}{c}\mathrm{Fe} \\
(\mathrm{mg} / 100 \mathrm{~g})\end{array}$ & $\begin{array}{c}\mathrm{Mn} \\
(\mathrm{mg} / 100 \mathrm{~g})\end{array}$ & $\begin{array}{c}\mathrm{Mg} \\
(\mathrm{mg} / 100 \mathrm{~g})\end{array}$ \\
\hline 33 & HM-277 & 103.73 & 138.27 & 1636.00 & 1.34 & 18.10 & 10.17 & 3.40 & 6.60 & 1.37 & 9.80 & 1.50 & 148.00 \\
\hline 34 & HM-277-1 & 104.13 & 135.53 & 1362.00 & 1.52 & 18.13 & 7.63 & 3.60 & 7.40 & 1.47 & 10.20 & 1.53 & 169.00 \\
\hline 35 & HM-273 & 110.10 & 135.50 & 1608.67 & 1.23 & 22.57 & 8.87 & 3.27 & 6.53 & 1.46 & 10.60 & 1.49 & 172.67 \\
\hline 36 & HM-260 & 98.17 & 156.30 & 1563.00 & 1.45 & 18.80 & 9.30 & 3.27 & 6.53 & 1.47 & 8.80 & 1.72 & 178.00 \\
\hline 37 & NDM-8 & 104.10 & 142.27 & 1376.00 & 1.35 & 18.60 & 8.93 & 3.27 & 6.60 & 2.34 & 10.20 & 1.59 & 184.00 \\
\hline 38 & NDM-7 & 107.20 & 143.23 & 1485.33 & 1.32 & 20.57 & 9.60 & 3.60 & 7.20 & 2.01 & 9.60 & 1.51 & 167.67 \\
\hline 39 & NDM-6 & 101.20 & 134.20 & 1452.00 & 1.34 & 21.50 & 9.39 & 3.40 & 7.20 & 2.38 & 10.60 & 1.36 & 167.00 \\
\hline 40 & NDM-5 & 105.27 & 143.53 & 1642.00 & 1.67 & 21.50 & 8.60 & 3.60 & 6.60 & 2.35 & 8.40 & 1.54 & 149.00 \\
\hline 41 & NDM-4 & 101.40 & 162.27 & 1595.33 & 1.45 & 21.20 & 8.80 & 3.60 & 6.60 & 2.25 & 9.93 & 1.56 & 192.00 \\
\hline 42 & NDM-10 & 104.50 & 150.30 & 1682.00 & 1.52 & 21.50 & 8.30 & 3.63 & 6.40 & 2.57 & 8.60 & 1.52 & 148.00 \\
\hline 43 & NDM-3 & 103.40 & 134.60 & 1456.00 & 1.28 & 22.60 & 8.60 & 3.63 & 6.60 & 2.12 & 8.87 & 1.56 & 184.00 \\
\hline 44 & NDM-2 & 104.60 & 141.30 & 1575.33 & 1.57 & 24.60 & 8.27 & 3.60 & 6.80 & 2.56 & 10.07 & 1.45 & 182.00 \\
\hline 45 & NDM-1 & 103.00 & 158.50 & 1359.33 & 1.45 & 19.60 & 8.60 & 3.60 & 6.40 & 2.24 & 9.13 & 1.43 & 167.67 \\
\hline 46 & NDM-25 & 104.40 & 134.50 & 1628.00 & 1.41 & 22.50 & 8.30 & 3.60 & 6.60 & 1.68 & 9.10 & 1.45 & 164.00 \\
\hline 47 & NDM-18 & 105.60 & 143.87 & 1384.00 & 1.69 & 20.40 & 9.60 & 3.40 & 6.60 & 1.74 & 9.40 & 1.54 & 179.00 \\
\hline 48 & NDM-19 & 95.97 & 138.07 & 1683.00 & 1.78 & 18.30 & 8.60 & 3.20 & 6.20 & 2.24 & 8.60 & 1.68 & 158.00 \\
\hline 49 & NDM-20 & 99.00 & 134.20 & 1527.00 & 1.56 & 20.60 & 8.40 & 3.40 & 6.60 & 1.46 & 10.20 & 1.47 & 183.00 \\
\hline 50 & NDM-26 & 105.30 & 148.33 & 1384.00 & 1.46 & 18.20 & 8.40 & 3.20 & 6.60 & 1.48 & 10.60 & 1.52 & 190.00 \\
\hline 51 & NDM-15 & 104.27 & 135.50 & 1750.00 & 1.78 & 19.80 & 9.60 & 3.50 & 6.60 & 2.34 & 9.40 & 1.50 & 175.00 \\
\hline 52 & NDM-14 & 98.60 & 138.40 & 1373.00 & 1.45 & 22.80 & 8.40 & 3.60 & 6.44 & 2.38 & 10.30 & 1.48 & 168.00 \\
\hline 53 & NDM-13 & 105.27 & 139.40 & 1456.00 & 1.34 & 18.60 & 9.40 & 3.60 & 6.47 & 2.45 & 9.60 & 1.62 & 168.00 \\
\hline 54 & NDM-21 & 108.43 & 137.37 & 1626.00 & 1.36 & 18.77 & 9.44 & 3.20 & 6.40 & 2.64 & 8.50 & 1.58 & 156.00 \\
\hline 55 & NDM-11 & 104.20 & 156.50 & 1628.00 & 1.76 & 20.60 & 8.73 & 3.40 & 6.40 & 1.48 & 10.60 & 1.35 & 183.00 \\
\hline 56 & NDM-12 & 108.27 & 135.50 & 1482.00 & 1.82 & 22.60 & 8.80 & 3.60 & 6.54 & 1.38 & 10.40 & 1.46 & 168.00 \\
\hline 57 & NDM-33 & 105.27 & 136.13 & 1627.00 & 1.34 & 24.63 & 9.44 & 3.20 & 6.00 & 1.37 & 10.20 & 1.52 & 156.00 \\
\hline 58 & NDM-24 & 103.17 & 134.60 & 1284.00 & 1.36 & 20.50 & 8.60 & 3.40 & 6.44 & 2.45 & 8.40 & 1.58 & 179.33 \\
\hline 59 & NDM-23 & 105.30 & 139.20 & 1626.00 & 1.67 & 21.73 & 8.40 & 3.60 & 7.20 & 2.67 & 9.40 & 1.50 & 149.00 \\
\hline 60 & NDM-22 & 107.27 & 134.50 & 1472.00 & 1.34 & 21.40 & 8.60 & 3.60 & 7.40 & 1.67 & 10.60 & 1.53 & 164.00 \\
\hline 61 & NDM-27 & 105.27 & 146.60 & 1536.00 & 1.34 & 22.40 & 8.50 & 3.60 & 6.44 & 1.45 & 10.80 & 1.46 & 174.00 \\
\hline 62 & NDM-32 & 108.30 & 135.60 & 1472.00 & 1.24 & 22.60 & 9.47 & 3.20 & 6.70 & 1.89 & 10.60 & 1.48 & 183.00 \\
\hline 63 & NDM-31 & 103.70 & 135.50 & 1528.00 & 1.35 & 22.80 & 8.60 & 3.20 & 6.60 & 1.65 & 9.47 & 1.62 & 185.00 \\
\hline 64 & NDM-28 & 105.30 & 126.60 & 1435.33 & 1.76 & 22.40 & 9.50 & 3.10 & 6.80 & 1.47 & 10.40 & 1.52 & 175.33 \\
\hline
\end{tabular}




\begin{tabular}{|c|c|c|c|c|c|c|c|c|c|c|c|c|c|}
\hline \multirow[b]{2}{*}{ S. No. } & \multirow[b]{2}{*}{ Genotypes } & \multirow{2}{*}{$\begin{array}{l}\text { Polyphenol } \\
\text { (mg/100 g) }\end{array}$} & \multirow[b]{2}{*}{$\begin{array}{l}\text { Phytic acid } \\
\text { (mg/100 g) }\end{array}$} & \multirow{2}{*}{$\begin{array}{l}\text { Saponin } \\
(\mathrm{mg} / 100 \mathrm{~g})\end{array}$} & \multirow{2}{*}{$\begin{array}{c}\text { Carbohydrate } \\
(\%)\end{array}$} & \multirow[b]{2}{*}{ Protein (\%) } & \multicolumn{7}{|c|}{ Proximate analysis } \\
\hline & & & & & & & $\begin{array}{c}\text { Moisture } \\
(\%)\end{array}$ & $\begin{array}{l}\text { Ash } \\
(\%)\end{array}$ & $\begin{array}{c}\text { Fiber } \\
(\mathrm{g} / 100 \mathrm{~g})\end{array}$ & $\begin{array}{c}\mathrm{Zn} \\
(\mathrm{mg} / 100 \mathrm{~g})\end{array}$ & $\begin{array}{c}\mathrm{Fe} \\
(\mathrm{mg} / 100 \mathrm{~g})\end{array}$ & $\begin{array}{c}\mathrm{Mn} \\
(\mathrm{mg} / 100 \mathrm{~g})\end{array}$ & $\begin{array}{c}\mathrm{Mg} \\
(\mathrm{mg} / 100 \mathrm{~g})\end{array}$ \\
\hline 65 & NDM-30 & 103.60 & 145.60 & 1542.00 & 1.56 & 22.60 & 9.70 & 3.60 & 6.40 & 2.34 & 8.60 & 1.69 & 183.00 \\
\hline 66 & NDM-29 & 106.13 & 134.50 & 1245.00 & 1.45 & 20.50 & 8.40 & 3.40 & 6.40 & 2.62 & 7.80 & 1.48 & 167.00 \\
\hline 67 & RM-33 & 105.27 & 138.30 & 1427.00 & 1.78 & 22.60 & 7.80 & 3.60 & 6.60 & 2.45 & 9.40 & 1.48 & 147.00 \\
\hline 68 & RM-27 & 105.20 & 156.67 & 1635.00 & 1.56 & 18.40 & 9.80 & 3.50 & 6.80 & 2.20 & 8.50 & 1.48 & 185.67 \\
\hline 69 & RM-195 & 102.27 & 134.40 & 1638.00 & 1.34 & 19.50 & 8.40 & 3.40 & 6.40 & 2.36 & 8.60 & 1.56 & 183.00 \\
\hline 70 & RM-187 & 106.30 & 158.37 & 1483.00 & 1.30 & 22.10 & 9.60 & 3.40 & 6.60 & 2.46 & 8.60 & 1.52 & 175.00 \\
\hline 71 & RM-185 & 104.20 & 135.40 & 1780.67 & 1.32 & 22.30 & 8.30 & 3.60 & 6.57 & 1.67 & 10.20 & 1.48 & 178.00 \\
\hline 72 & RM-16 & 106.27 & 134.20 & 1568.00 & 1.56 & 22.60 & 8.40 & 3.60 & 7.20 & 1.58 & 10.40 & 1.48 & 158.00 \\
\hline 73 & RM-190 & 106.20 & 162.40 & 1378.00 & 1.78 & 22.60 & 8.60 & 3.40 & 6.30 & 2.48 & 8.50 & 1.56 & 197.00 \\
\hline 74 & RM-14 & 108.20 & 165.27 & 1652.00 & 1.34 & 22.30 & 8.50 & 3.20 & 6.40 & 2.68 & 8.40 & 1.46 & 148.00 \\
\hline 75 & RM-15 & 104.30 & 154.60 & 1682.00 & 1.45 & 22.50 & 8.60 & 3.40 & 6.80 & 2.48 & 9.40 & 1.41 & 185.00 \\
\hline 76 & RM-18 & 105.30 & 105.30 & 1634.33 & 1.52 & 18.40 & 8.57 & 3.60 & 6.80 & 1.68 & 10.20 & 1.58 & 162.00 \\
\hline 77 & RM-13 & 106.42 & 105.73 & 1578.00 & 1.36 & 18.30 & 9.30 & 3.20 & 6.40 & 2.34 & 8.40 & 1.52 & 154.00 \\
\hline 78 & RM-186 & 107.30 & 105.30 & 1583.00 & 1.68 & 18.63 & 8.47 & 3.60 & 6.40 & 2.56 & 7.77 & 1.48 & 165.00 \\
\hline 79 & RM-199 & 103.20 & 102.20 & 1653.00 & 1.56 & 19.50 & 8.63 & 3.70 & 7.40 & 2.50 & 8.20 & 1.64 & 184.00 \\
\hline 80 & RM-189 & 105.30 & 105.30 & 1738.00 & 1.45 & 18.50 & 9.83 & 3.46 & 6.30 & 2.78 & 8.27 & 1.72 & 149.00 \\
\hline 81 & RM-70 & 102.40 & 104.33 & 1527.00 & 1.34 & 18.17 & 9.63 & 3.40 & 6.70 & 2.34 & 8.20 & 1.50 & 157.00 \\
\hline 82 & RM-28 & 104.50 & 138.40 & 1374.00 & 1.45 & 18.20 & 8.80 & 3.40 & 6.60 & 1.55 & 10.30 & 1.48 & 184.00 \\
\hline 83 & RM-424 & 105.20 & 125.50 & 1384.00 & 1.34 & 22.40 & 9.87 & 3.60 & 6.38 & 1.67 & 10.20 & 1.58 & 168.00 \\
\hline 84 & RM-198 & 104.20 & 137.93 & 1546.00 & 1.78 & 22.10 & 9.77 & 3.60 & 6.60 & 1.28 & 10.87 & 1.55 & 174.00 \\
\hline 85 & RM-10 & 105.60 & 146.40 & 1467.00 & 1.60 & 20.60 & 8.53 & 3.40 & 6.40 & 2.34 & 9.60 & 1.52 & 194.00 \\
\hline 86 & ACC-002 & 104.60 & 158.70 & 1652.00 & 1.45 & 19.13 & 8.67 & 3.40 & 6.40 & 2.55 & 9.20 & 1.68 & 158.00 \\
\hline 87 & ACC-001 & 103.60 & 134.20 & 1582.00 & 1.37 & 22.40 & 8.83 & 3.40 & 6.47 & 2.45 & 7.77 & 1.58 & 168.00 \\
\hline 88 & ACC-003 & 103.54 & 162.33 & 1496.00 & 1.35 & 19.80 & 8.83 & 3.80 & 6.20 & 1.47 & 10.47 & 1.59 & 186.00 \\
\hline 89 & ACC-017 & 105.53 & 164.20 & 1375.00 & 1.34 & 22.60 & 9.77 & 3.40 & 6.37 & 2.67 & 9.13 & 1.46 & 175.00 \\
\hline 90 & ACC-010 & 104.27 & 126.27 & 1632.00 & 1.32 & 22.80 & 9.87 & 3.60 & 6.60 & 2.56 & 8.31 & 1.62 & 189.00 \\
\hline 91 & ACC-009 & 105.50 & 156.37 & 1736.00 & 1.35 & 21.30 & 8.53 & 3.13 & 6.50 & 1.82 & 9.13 & 1.48 & 168.00 \\
\hline 92 & ACC-006 & 107.30 & 147.30 & 1655.33 & 1.67 & 22.80 & 9.50 & 3.60 & 6.37 & 1.48 & 10.03 & 1.59 & 172.00 \\
\hline 93 & ACC-004 & 99.47 & 148.17 & 1637.00 & 1.34 & 20.97 & 9.67 & 3.60 & 6.20 & 1.39 & 10.27 & 1.48 & 189.00 \\
\hline 94 & ACC-012 & 110.30 & 176.30 & 1254.00 & 1.82 & 20.77 & 8.37 & 3.70 & 6.17 & 1.44 & 10.60 & 1.44 & 180.00 \\
\hline 95 & ACC-007 & 100.40 & 154.30 & 1453.33 & 1.56 & 20.73 & 9.30 & 3.20 & 6.53 & 1.68 & 8.67 & 1.43 & 143.00 \\
\hline 96 & ACC-013 & 105.80 & 167.31 & 1462.00 & 1.46 & 21.40 & 8.57 & 3.60 & 6.57 & 1.93 & 8.13 & 1.48 & 165.00 \\
\hline
\end{tabular}




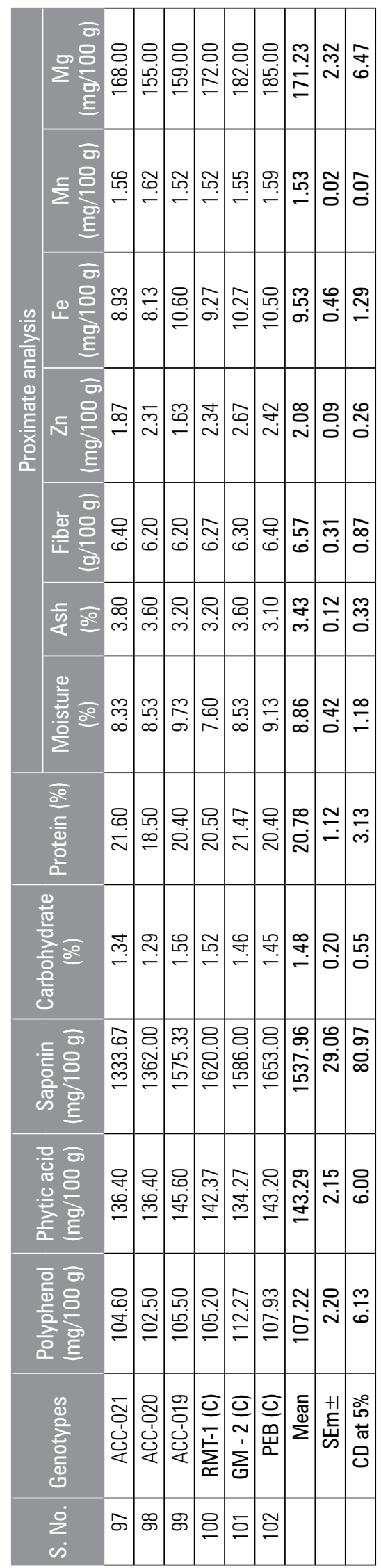

The carbohydrate content in seeds ranged from 1.07 to $1.82 \%$ with an overall mean of $1.48 \%$. It was observed to be the maximum in seeds of genotypes NDM-12 (1.82\%) and ACC-012 (1.82\%) followed by UM-117 (1.81\%), whereas, its minimum value was recorded in genotype UM-120 (1.07\%).

Protein content in seeds varied from 18.1 to $24.63 \%$ with an average mean of 20.78 per cent. The maximum protein content in seeds was exhibited in genotype NDM -33 (24.63\%) while, it was minimum in HM-258-1 (18.1\%).

The mean moisture content in seeds was recorded to be $8.86 \%$ and it ranged from 7.57 to $11.51 \%$. The maximum moisture per cent was observed in genotype HM-258-1 (11.51\%), whereas, the minimum in genotype UM-130 (7.57\%).

Ash content in seed lied between 3.0 to $3.87 \%$ with an overall mean of $3.43 \%$. The highest ash content was recorded in genotype UM-132 (3.87\%) while, the minimum (3.0\%) was observed in genotype UM-114 and was preceded by NDM-28 (3.1\%).

Fiber content varied from 5.6 to $8.93 \mathrm{~g} / 100 \mathrm{~g}$, with a general mean $6.57 \mathrm{~g} / 100 \mathrm{~g}$. Genotype UM-113 recorded the utmost ( $8.93 \mathrm{~g} / 100 \mathrm{~g}$ ) fiber content however; it was the least in genotype UM-114 (5.6 g/100 g).

The content of zinc ranged from 1.28 to 2.78 $\mathrm{mg} / 100 \mathrm{~g}$ with an average of $2.08 \mathrm{mg} / 100 \mathrm{~g}$. It was the maximum in RM-189 (2.78 $\mathrm{mg} / 100$ g) whereas, the minimum in RM-198 (1.28 $\mathrm{mg} / 100 \mathrm{~g})$.

Iron content in seed varied from $7.77 \mathrm{mg} / 100 \mathrm{~g}$ to $11.2 \mathrm{mg} / 100 \mathrm{~g}$, with a mean of $9.53 \mathrm{mg} / 100$ g. The maximum iron content was recovered in the genotype HM-271 (11.2 mg/100 g), while, ACC-001 and RM-186 recorded the least content $(7.77 \mathrm{mg} / 100 \mathrm{~g})$. 
Manganese content ranged from $1.79 \mathrm{mg} / 100$ $\mathrm{g}$ to $1.35 \mathrm{mg} / 100 \mathrm{~g}$, with an average of 1.53 $\mathrm{mg} / 100 \mathrm{~g}$. Maximum content exhibited in genotype UM-116 (1.79 mg/100 g) however; NDM11 recorded the minimum content in seed $(1.35$ $\mathrm{mg} / 100 \mathrm{~g})$.

The content of magnesium in seed varied from $143 \mathrm{mg} / 100 \mathrm{~g}$ to $197 \mathrm{mg} / 100 \mathrm{~g}$, with an overall mean performance of $171 \mathrm{mg} / 100 \mathrm{~g}$. Genotype RM-190 observed the highest magnesium content $(197 \mathrm{mg} / 100 \mathrm{~g})$ whereas, ACC-007 recorded the lowest content (143 mg/100 g).

\section{DISCUSSION}

The antioxidant activity could be correlated with the polyphenolic components present in the extract of fenugreek seeds. The polyphenolic compounds of fenugreek seeds can be considered cytoprotective during $\mathrm{EtOH}$-induced liver damage. Polyphenolic flavonoids have been shown to protect various cell types from oxidative stress-mediated cell injury. These results were in harmony with the findings of Gupta and Singh (2002). It was also observed that polyphenols contents in fenugreek depend not only on the genotype but also on the degree of its environment threats (temperature, light etc). Avtar et al. (2003) reported that the levels of TP (total phenol) and some of their activities increased in response to infection and decreased at higher disease severity levels.

Gupta and Singh (2002) investigated the changes in anti-nutritional factors at growth stages of fenugreek leaves of four genotypes and found that TP and flavonols increased with growth of leaves (leaf age) in two genotypes while they increased up to the second cutting and decreased thereafter in the others genotypes.

The abundance of phytic acid in cereal grains is a concern in the foods industries because the phosphorus in this form is unavailable to monogastric animals due to a lack of endogenous phytases; enzymes specific for the dephosphorylation of phytic acid (Asada et al., 1969). Phytic acid is the major contributor to reduced bioavailability of $\mathrm{Fe}$ and $\mathrm{Zn}$ in fenugreek and other legume crops. However, very little is known about how much of the seed Fe and $\mathrm{Zn}$ is bioavailable for absorption. Welch et al. (2000) detected large differences in Fe bioavailability ranging from $53 \%$ to $76 \%$ of total Fe, with higher seed Fe genotypes resulting in increased amount of total bioavailable Fe.

Diosgenin, a naturally-occurring steroid saponin is found abundantly in fenugreek. It is a precursor of various synthetic steroidal drugs that are extensively used in the pharmaceutical industry. The results were in harmony with the findings of Cerdon et al. (1995). Double pod characteristics is linked to high saponin and diosgenin content, it is expected that some of these mutants may produce more saponin and diosgenin in addition to producing high seed yield (Acharya et al., 2006). High saponin producing lines are mostly preferred by the nutraceutical industry.

The results relegated with the carbohydrate content in seed are in consonance with Kochhar et al. (2006) and Sumayya et al. (2012).

Fenugreek seed was reported to be rich in protein with a well balanced amino acid pattern. This could probably be attributed to the increase in $\mathrm{N}_{2}$-fixing efficiency of inoculated plants where more nitrogen was fixed and translocated to the seed. Moreover, nodulation enhanced the symbiotic properties of fenugreek plants and better growth and production were obtained by inoculation. The results were in harmony with the findings of Kochhar et al. (2006), Singh et al. (2010), and Sumayya et al. (2012).

The moisture content in seeds is affected by the relative humidity of the surrounding atmo- 
sphere at the time of harvest and during storage. The present findings are in consonance with Gopalan et al. (1992) and Kochhar et al. (2006).

\section{Proximate analysis}

The fenugreek seeds are rich in leucine, valine, lysine, and phenylalanine. Phytochemicals in fenugreek vary depending not only on the genotype of the plant but also on the environment under which it is grown. Plants use these compounds to defend themselves against various threats of nature (biotic and abiotic) and levels of such resistant chemicals were found to change as plants become more mature.

Ash is the substance that remains after burning an organic substance; it contains almost all macro- as well as micronutrients except organic carbon and nitrogen. The findings of the present study are quite similar to that of Gopalan et al. (1992).

The content of zinc ranged from 1.28 to 2.78 $\mathrm{mg} / 100 \mathrm{~g}$ with an average of $2.08 \mathrm{mg} / 100 \mathrm{~g}$. The results were in harmony with the Rao and Deosthale (1981) and Abd El-Aal and Rahma (1986).

In India, over 9 million disability adjusted life years (DALYs) are lost annually due to iron, zinc and other micronutrient deficiency, with iron deficiency alone contributing to 4 million DALYs lost (Qaim et al., 2007). The disease burden associated with iron deficiency in India could be reduced by 19-58 per cent by crop biofortification (Stein et al., 2008). Fenugreek could act as a cheaper and one of the most effective measures against DALY's and may contribute towards crop biofortification. Meenakshi et al. (2010) concluded that overall biofortification can make a significant impact on reducing the burden of micronutrient deficiencies in the developing world in a highly cost effective manner; however the impact differ depending on the combination of crop, micronutrient and country, and the major reasons underlying these differences are identified to inform policy. However, these approaches have had only limited success and could not by themselves attain sufficiently millennium development goals mainly because sub interventations require infrastructure, continues flow of resources, purchasing power or access to markets and health care systems to their success, often not available to people living in remote areas. The rural based diets are predominantly composed of cereals and legumes. Fenugreek being a nutritious leafy legume crop could thus be a better option for overcoming such adverse situations.

Integrating micronutrient rich foods, such as legumes and vegetables, into diets is the most practical and sustainable way to alleviate micronutrient deficiency. A final issue of utmost importance is adoption of biofortified cultivars of fenugreek. The farmers perception of adoptability of a new cultivars must be taken into account and such that new cultivars should show yield superiority produce more than the existing cultivars with respect to seed yield and other characteristics such as taste, size, color, and flavour. For biofortified crops, there should be assured market and the produce must earn farmers more income if the cultivars widely adopted and, therefore, the nutritional benefit is to be widespread. Consumer acceptance of the biofortified crops could be an issue if the new interventation changes the appearance or taste of the crop however; this may not be the case for the crops biofortified with so called "invisible traits" such as iron, zinc and others. This paper focused on the progress realized towards developing seed nutrient dense ( $\mathrm{Fe}, \mathrm{Mg}, \mathrm{Zn}$ and $\mathrm{Mn}$ ) genotypes of fenugreek, by exploiting natural genetic variation using conventional breeding.

In addition, soil and environmental factors, especially soil water regime and climatic factors, such as temperatures, also influence yield and the seed micronutrient quality relative to $\mathrm{Fe}$ 
and Zn composition (Stewart et al., 2005). Since seed $\mathrm{Fe}, \mathrm{Zn}$ and other micronutrients are liable to contamination during harvest and preparation of the samples for analysis. Polyphenols in foods may chelate dietary Fe and lower its bioavailability (Siow et al., 2008). Natural variation in plant genetic resources provides the basic raw material and plays a fundamental role in crop improvement programs.

The environmental variables, such as soil $\mathrm{pH}$, temperature, solar radiation, precipitation, organic matter, and soil texture, have the potential to influence nutrient concentration and must be taken into consideration while explaining the variation for plant micronutrient in germplasm or when assessing the nutritional quality of staple food crops grown in diverse agro ecological conditions. More studies are needed to assess the efficacy of biofortified products in raising micronutrient status in human subjects. Biofortification often alters the flavor, taste, appearance, and other features of the foods, which may limit the consumer acceptance - an issue that merits further investigation. The biofortified crops must be adopted by farmers and consumed by those suffering from micronutrient malnutrition.

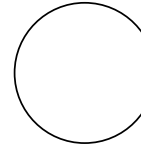

Abd El-Aal, M.H. and E.H. Rahma. 1986. Changes in gross chemical composition with emphasis on lipid and protein fractions during germination of fenugreek seeds. Food Techn. 9 (2), 53-59.

Acharya, S., A. Srichamroen, S. Basu, B. Ooraikul, and T. Basu. 2006. Improvement in the nutraceutical properties of fenugreek (Trigonella foenum-graecum L.) Songklanakarin J. Sci. Technol. 28 (Suppl. 1), 1-9.

AOAC 1995. Official methods of analysis. Association of Official Analytical Chemists, Washington D.C.

Asada, K. K., Tanaka, and Z. Kasai. 1969. Formation of phytic acid in cereal grains. Ann. N.Y. Acad. Sci. $165,801-814$.

Avtar, R., A.S., Rathi, D.S. Jatasra, and U.N. Joshi. 2003. Changes in phenolics and some oxidative enzymes in fenugreek leaves due to powdery mildew infection. Acta Phytopathologica et Entomologica Hungarica 38(3-4), 237-244.

Budavari, S. 1996. The merck index: An encyclopedia of chemicals, drugs, and biological. $12^{\text {th }}$ ed. N.J. Merk \& Co, Inc., Whitehouse Station, NJ.

Cerdon, C., A. Rahier, M. Taton, and Y. Sauvaire. 1995. Effects of tetcyclacis on growth and on sterol and sapogenin content in fenugreek. J. Plant Growth Regul. 14(1), 15-22.

Cochran, G.W. and G.M. Cox 1950. Experimental designs. John Wiley and Sons, New York, NY.

\section{BIBLIOGRAPHIC REFERENCES}

Fisher, R.A. and F. Yates 1963. Statistical tables for biological, agricultural and medical research. Oliver and Boyd, London.

Gopalan, C., B.V. Rama Sastri, and S.C. Balasubramanian. 1992. Nutritive value of Indian Foods. NIN, ICMR, Hyderabad 19, 13-22.

Gupta, K. and J. Singh. 2002. Anti-nutritional and flatulence factors at various stages of vegetative growth of fenugreek (Trigonella foenum graecum L.) leaves. J. Food Sci. Technol. 39(5), 525-527.

Hedge, J.E. and B.T. Hofreiter. 1962. Methods of estimating starch and carbohydrates. Pp. 163-201. In: Whistler, R.L. and Be Miller, J.N. (eds.). Carbohydrate chemistry 17. Academic Press, New York, NY.

Kochhar, A., M. Nagi, and R. Sachdeva. 2006. Proximate composition, available carbohydrates, dietary fibre and anti nutritional factors of selected traditional medicinal plants. J. Hum. Ecol. 19(3), 195-199.

Lin, J.T. and D.J. Yang. 2008. Determination of steroidal saponins in different organs of yam (Dioscorea pseudojaponica Yamamoto). Food Chem. 1089, 1608-1674.

Lowry, O.H., N.J. Rosebrough, A.L. Farr, and R.J. Randall. 1951. Protein measurement with Folin reagent. J. Biol. Chem. 193, 265-273.

Malik, C.P. and M.B. Singh, M.B. 1980. Peroxidase. p. 286. In: Plant enzymology and histo enzymology. Kalyani Publisher, New Delhi, India. 
Max, B. 1992. This and that - the essential pharmacology of herbs and spices. Trends Pharmacol. Sci. 13, 15-20.

Meenakshi, J.V., N.L. Johnson, V.M. Manyong, H. DeGroote, J. Javelosa, D.R. Yanggen, F. Naher, C. Gonzalez, J. García, and E. Meng. 2010. How costeffective is biofortificationin combating micronutrient malnutrition. An ex ante assessment. World Development 38(1), 64-75.

Mehrafarin, A., A. Qaderi, S. Rezazadeh, H. Naghdibadi, G. Noormohammadi, and E. Zand. 2010. Bioengineering of important secondary metabolites and metabolic pathways in fenugreek (Trigonella foenum graecum L.). J. Medicinal Plants 9(35), 1-18.

Nasroallah, M. and M. Kolsum. 2013. Physiological and pharmaceutical effects of fenugreek (Trigonella foenum-graecum L.) as a multipurpose and valuable medicinal plant. Global J. Medicinal Plant Res. 1(2), 199-206.

Newall, C.A., L.A. Anderson, and J.D. Phillipson. 1996. Herbal medicines: A guide for healthcare professionals. The Pharmaceutical Press, London.

Qaim, M. A., J. Stein, and J.V. Meenakshi. 2007. Economics of biofortification. Agric. Econ. 37, 119-133.

Rao, D.S.S, and Y.G. Deosthale. 1981. Mineral composition of four Indian food legumes. J. Food Sci. 46, 1962-1963.
Singh, P., U. Singh, M. Shukla, and R.L. Singh. 2010. Variation of some phytochemicals in methi and saunf plants at different stages of development. J. Herbal Medicine Toxicol. 4(2), 93-99.

Siow Y.T., K.Y. Chi, T. Elad, P.G. Raymond, R.M. Welch, L. Xingen, and D.D. Miller. 2008. Iron bioavailability to piglets from red and white common beans (Phaseolus vulgaris). J. Agric. Food Chem., 56 (13), pp 5008-5014.

Stein, A.J., J.V. Meenakshi, and M. Qaim. 2008. Potential impacts of iron biofortification in India. Social Sci. Medicine 66(8), 1797-1808.

Stewart, W.M., D.W. Dibb, A.E. Johnston, and T.J. Smyth. 2005. The contribution of commercial fertilizer nutrients to food production. Agron. J. 97, 1-6.

Sumayya, A.R., S. Sivagami, and A. Nabeelah. 2012. Screening and biochemical quantification of phytochemicals in fenugreek (Trigonella foenum-graecum). Res. J. Pharm. Biol. Chem. Sci. 3(1), 165-169.

Welch, R.M. 2001. Micronutrients, agriculture and nutrition; linkages for improved health and well being. Science Publisher, Jodhpur, India. pp. $247-$ 289.

Wheeler, E.L. and R.E. Ferrel. 1971. A method for phytic acid determination in wheat and wheat fractions. Cereal Chem. 48, 312-320. 